\title{
Survey of cost models for space telescopes
}

\author{
H. Philip Stahl, FELLOW SPIE \\ NASA Marshall Space Flight Center \\ P.O. Box 9262 \\ Huntsville, Alabama 35812 \\ E-mail: H.Philip.Stahl@nasa.gov
}

\begin{abstract}
Parametric cost models are routinely used to plan missions, compare concepts, and justify technology investments. However, great care is required. There is a lot of confusion and wrong information for space telescopes. Cost estimating relationships based on primary mirror diameter vary by an order of magnitude. Cost estimating relationships based only on mass lack sufficient detail to support concept analysis and can lead to inaccurate conclusions by encouraging excessively complex and technologically immature solutions. Similarly, using ground-based models leads to incorrect conclusions. This work surveys current and historical published cost models for space telescopes while attempting to interpret them in a common logical framework to enable a systematic intercomparison. () 2010 Society of Photo-Optical Instrumentation Engineers. [DOI: 10.1117/1.3430603]
\end{abstract}

Subject terms: space telescope cost model; parametric cost model; cost model.

Paper 090900PR received Nov. 15, 2009; revised manuscript received Feb. 22 2010; accepted for publication Mar. 26, 2010; published online May 28, 2010.

\section{Introduction}

Parametric cost models for space telescopes have several uses. They identify major architectural cost drivers and allow high-level design trades. They enable cost-benefit analysis for technology development investment. And, they provide a basis for estimating total project cost used to justify the initiation of billion dollar programs. However, great care is required. All cost models are wrong and should be used with healthy skepticism. And, while some models are useful, others are really wrong. This work reviews and summarizes historical and current published cost models for space telescopes. Each model is presented using its original nomenclature and definitions. Whenever possible, the work attempts to place models into a present context while intercomparing models and other relevant information. This work does not include unpublished proprietary models or models developed to study specific mission concepts.

\section{Cost Models}

\subsection{Ground Telescope Cost Models}

Cost models for ground-based telescopes have been published since the 1960s. However, at the time, there was insufficient data to generate similar cost models for space telescopes. In the absence of a space telescope cost model, the community naturally relied on what it knew and extrapolated based on ground telescope models. Ground telescope cost models historically focused on primary mirror diameter as the principal cost driver. A detailed discussion of ground cost models can be found in Stahl, 2005. ${ }^{1}$

Starting in the 1960s, models scaled cost as a function of primary mirror diameter raised to the power of 3.0. Since that time, many different scaling laws have been proposed by different and, in some cases, even the same author. In his 1979 paper, Meinel ${ }^{2}$ found a "scaling law exponent

0091-3286/2010/\$25.00 @ 2010 SPIE close to the 2.0 power, in contrast to the often cited 2.7 exponent." However, in later papers, ${ }^{3,4}$ he reported scaling laws ranging from 2.5 to 2.75 . Of all these laws, the one which seems to have gained the most acceptance is the 2.7 factor. In 2000, Bely indicated that a scaling law of 2.7 is considered the standard for ground-based observatories, and in 2002 Stepp stated that the consensus traditional scaling law appears to be $2.7 .^{6}$ While these laws are technically correct, they should not be extended to space telescopes, because they all include the ground observatory building and/or dome (the cost of the dome is driven by the volume of the telescope, which is proportional to $D$ raised to the third power). And, space telescopes do not have domes. Therefore, any use of a 2.7 scaling law for space telescopes will result in unfounded conclusions. Meinel actually made the dome distinction in his 1979 paper, but then failed to repeat this clarification in his later papers.

In 2005, Stahl $^{1}$ published a multivariable parametric cost model for just the ground telescope assembly (without telescope mount or dome), where the cost scales with primary mirror diameter to the 1.8 th power:

ground OTA cost $\$ \sim(S F)(D)^{1.8}(\lambda)^{-0.5} \exp [-0.04(Y)]$,

where $D$ is the primary mirror diameter, $\lambda$ is the wavelength diffraction-limited performance, $Y$ is the year of development for reduction in technology cost over time, and $S F$ is the segmentation factor.

$$
\begin{aligned}
S F= & P_{n} R_{n}^{0.7}(D S / D)^{1.8} \text { if the primary mirror is } \\
& \text { segmented and } \\
S F= & 1 \text { if the primary mirror is monolithic } \\
D s= & \text { segment diameter } \\
P_{n}= & \text { number of unique segment prescriptions } \\
R_{n}= & \text { number of repeated segments. }
\end{aligned}
$$

The segmentation factor estimates the cost reduction that can be gained by incorporating replication and serial processing learning into the fabrication of large telescopes. 
However, please note that this cost efficiency is not fully obtained if multiple parallel manufacturing lines are used.

The Stahl 2005 study hoped that a cost relationships for ground optical telescopes, without their domes, would be indicative of space optical telescope assembly costs. Unfortunately, as is discussed in Stahl et al. $2010{ }^{7}$ it is not. The cost to manufacture ground telescopes is not a good estimator of the cost to manufacture space telescopes.

\subsection{Space Telescope Cost Models}

Parametric cost models for space telescopes have not been published as much as for ground telescopes. This is partly because of the lack of an extensive database, i.e., there are not as many space telescopes, and partly because cost information for space telescopes is difficult to obtain either for proprietary or national security reasons. But, there have been some discussions.

\subsubsection{Meinel models}

In 1986, Meinel and Meinel asserted that, "Space telescopes are intrinsically 2 orders of magnitude more expensive for a given aperture than are terrestrial ones and are likely to remain at least 1 order of magnitude more expensive." ${ }^{\prime}$ However, it does not appear that this predicted cost reduction has occurred. Ten years later in 1997, Schmidt-Kaler and Rucks asserted that space telescopes were still more expensive than ground telescopes by a factor of almost $100 .^{9}$ These papers implied that space telescope cost scales with aperture diameter to either the 2.0 or 2.7 power. The Meinels revisited the subject of cost models for space telescopes in 2004 with lead author Bellea. ${ }^{10}$ At one point in the paper, they assert that, "no general inference can be drawn from the relationship between telescope cost and aperture size ... telescope size is independent of cost. Instead, our assessment is that the predominant phenomenon at play is rapid technological development." But, later in the paper and in the conclusion, they assert that it is their expectation that the scaling law for space-based telescopes is close to $D^{2.0}$. They make this argument based on a scaling of the structure necessary to maintain optical surface figure in a zero-gravity environment and the scaling of structure necessary to protect a space telescope from space weather.

\subsubsection{Bely models}

In 2000, Bely asserted that from his experience with "mostly classified systems cost data," that the scaling law for space telescopes based on primary mirror diameter is "on the order of 1.8th power." In his 2003 book, Bely published an equation for cost scaling with diameter to the 1.6th power. He justified this assertion based on the argument that space telescopes do not have a dome which scales with volume; and, that the cost to design and test a telescope is larger for a space than a ground telescope. ${ }^{11}$ But most importantly, Bely provided a citation for his equation: "For space telescopes, one model developed by Technomics, based for the most part on military and surveillance missions, is of the form:"
Cost $\propto \frac{D^{1.6} M_{f} D_{f} D_{f}^{\prime}}{\lambda^{1.8} T^{0.2} \exp [0.033(Y-1960)]}$,

where $D$ is diameter, the $M_{f}$ and $D_{f}$ terms are material and design factors, $T$ is temperature, and $Y$ is year. These parameters $\left(D, M_{f}, D_{f}, T\right.$, and $\left.Y\right)$ are all directly traceable to the Technomics citation provided by Bely, which is identical to Ref. 12 by Horak et al., 1993.

The Horak cost model is summarized later in this work and thus is not discussed here. However, the cost relationship reported by Bely is not the same as reported by Horak. There are three specific differences. Missing from the Bely equation is a term for the number of curved elements included in the Horak model. The Horak model exponent for wavelength is 0.178 not 1.8 - possibly a typo on the part of Bely. And, the exponent for aperture diameter in the cited Horak publication is 0.705 not 1.6. It is this last discrepancy which is of most interest to this author, because, during his tenure in industry, he saw several proprietary cost models with the same 1.6th power aperture diameter exponent. All of these models claimed to have been derived from the Horak model.

\subsubsection{Mass models}

In the space industry, mass is believed to be the key cost driver. Thus, the original NASA Air Force Cost Model (NAFCOM) estimated space mission cost based solely on mass. The problem is that a mass-only model lacks sufficient detail to make meaningful mission concept design trades. To correct this deficiency, since 2002 NAFCOM has incorporated cost estimating relationships (CERs) of heritage, technology readiness level (TRL), and other technical and programmatic parameters. NAFCOM has an extensive database containing parametric cost data and technical information at the group, subsystem, and component level for many historical NASA programs. These data are normalized and separated by mission type. In addition, NAFCOM contains data from the Scientific Instrument Cost Model (SICM). NAFCOM and SICM are computer software applications that calculate cost predictions based on user parameter inputs.

One example of a space telescope cost versus mass model is the NASA Advanced Mission Cost Model: ${ }^{13}$

$$
\begin{aligned}
\operatorname{cost}(F Y 04)= & \$ 2.25 \mathrm{~B}(\text { mass } / 10,000 \mathrm{~kg})^{0.654} \\
& \times\left(1.555^{\text {difficulty level }}\right) \times\left(N^{-0.406}\right),
\end{aligned}
$$

where $N$ is the number of flight systems; and difficulty levels are: $-2=$ very low, $-1=$ low, $0=$ average, $1=$ high, and $2=$ very high.

While it may initially appear, from the parametric equation, that mission cost is driven by mass, Fig. 1 shows that difficulty level is a larger cost driver than mass. To illustrate this fact, Fig. 1 has phase A to D cost data points for Hubble Space Telescope (HST), James Webb Space Telescope (JWST), and Kepler. While HST is nearly two times more massive than JWST, HST is less expensive because it is less difficult. Also, the model includes a cost reduction for multiple systems, but this author knows of no way to test this CER. Most, if not all, scientific space telescopes are "one-off" systems with very little duplication or even reuse. The only relevant examples of quantity production 


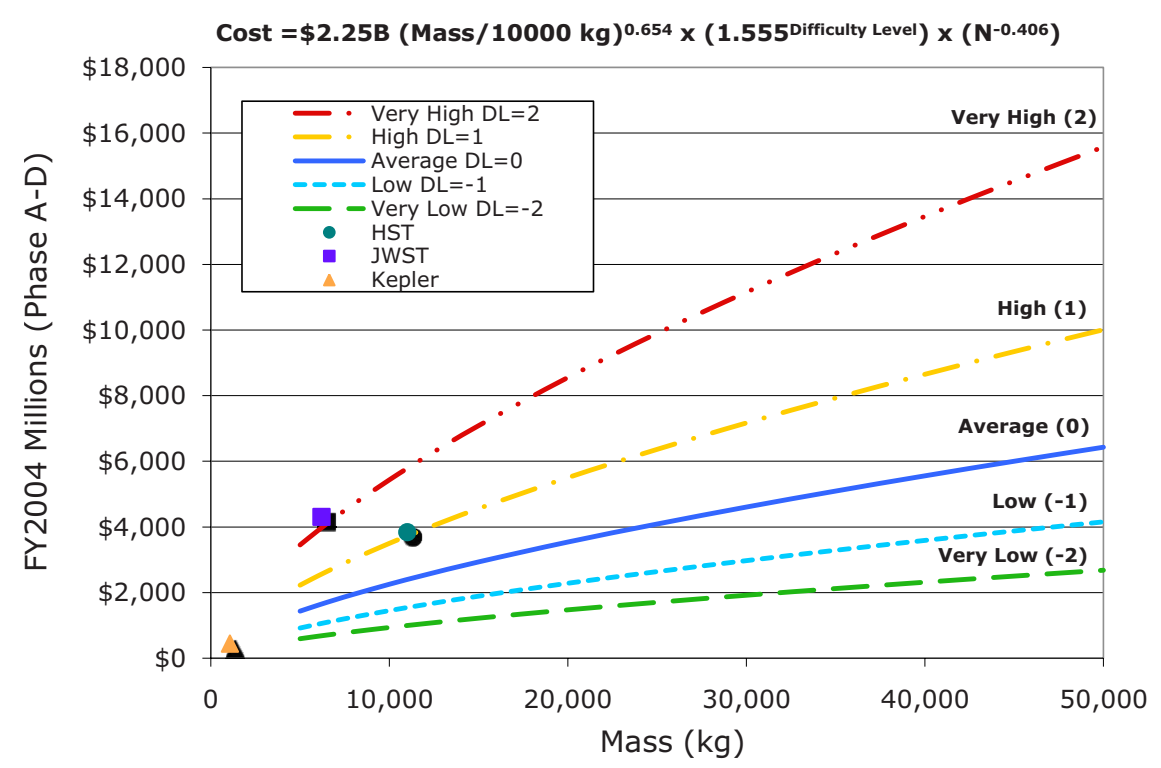

Fig. 1 The NASA Advanced Mission Cost Model ${ }^{13}$ estimates optical telescope cost based on mission mass, difficulty level, and quantity. While the parametric equation might emphasize mass as the dominant mission cost driver, difficulty level might be a larger cost driver than mass. The model reports cost in 2004 US dollars. Plotted on the model are phase A to D costs in FY09 dollars for HST, JWST, and Kepler. HST data comes from the REDSTAR database. JWST cost is from NASA FY10 budget, and Kepler cost is from NASA FY09 budget.

are commercial imaging systems such as IKONOS (Lockhead Martin, Bethesda, Maryland), Quickbird (Satellite Imaging Corporation, Houston, Texas), or NextView (Digital Globe, Longmont, Colorado), and their cost data is proprietary.

It is this author's opinion that there are two problems with mass models. First, mass is not a primary cost driver, but rather an indicator of the true cost drivers. And second, some may be tempted to focus only on mass and ignore not only the true engineering cost drivers (such as aperture) but also the critical role that complexity plays. A simplistic example as to why one cannot consider only mass is the lead brick analogy. It is silly to think that it costs more to launch a lead brick than a complex instrument with half the mass. In real life, mission planners are given a mass budget based on the capacities of the intended launch vehicle. Then they proceed to design their mission to this mass budget. The more functionality they include for a fixed mass, the more complex and more expensive the mission becomes.

\subsubsection{Planning Research Corporation cost models}

Planning Research Corporation (PRC) has generated several telescope cost models. In 1985, PRC published a model that predicts costs of telescopes for unmanned and manned spacecraft. ${ }^{14}$ The paper reports two cost estimating relationships: telescope design and development (D and D) and telescope flight hardware. These cost estimating relationships "represent the cost of a second unit following the manufacture and assembly of a prototype article. System level costs are included [for example: Systems Test Hardware (prototype); Systems Test Operations, GSE, Systems Engineering and Integration and Program Management]." PRC studied the parameters of weight, volume, primary mirror diameter, and minimum temperature. The PRC diameter only CER estimates $58 \%$ of total space telescope D and D and flight cost. For all space telescopes in their database (UV, visible, IR): design and development cost is proportional to $D^{0.276}$, flight unit cost is proportional to $D^{0.286}$, and D and D cost is approximately three times the flight unit cost. For just the infrared telescopes in their database: design and development cost is proportional to $D^{0.4782}$, flight unit cost is proportional to $D^{0.5576}$, and D and $\mathrm{D}$ cost is approximately four times the flight unit cost.

In 2000, Smart developed a multivariable parametric cost model: ${ }^{15}$

cost

$$
=\$ 521.967 M * M D^{1.120} * T R L^{-0.881} * A P^{0.187} * Y T^{-0.330},
$$

where $M D$ is the mirror diameter (meters), TRL is the technology readiness level, $A P$ is the average power (watt), and $Y T$ is the year of technology.

While Smart found that mirror diameter was the primary cost driver, it was not the only parameter. It was necessary to include other engineering parameters to account for variations in cost not related to mirror diameter. The more mature the technology used on a mission, the lower the cost. The more power required by a mission for its instruments, spacecraft, and data transmission, the higher the cost. And because technology investment reduces cost, the more recent the mission launch date, the lower its cost. This model was developed using 13 space telescopes: EUVE, HEAO-2 (Einstein), HST, SIRTF (Spitzer), TRACE, WIRE, IRAS, IUE, OAO-2, OAO-3 (Copernicus), Skylab 1 , and two Spacelab-2 missions. The regression statistics for this model explained $89 \%$ of the cost variation of these missions (Fig. 2). 


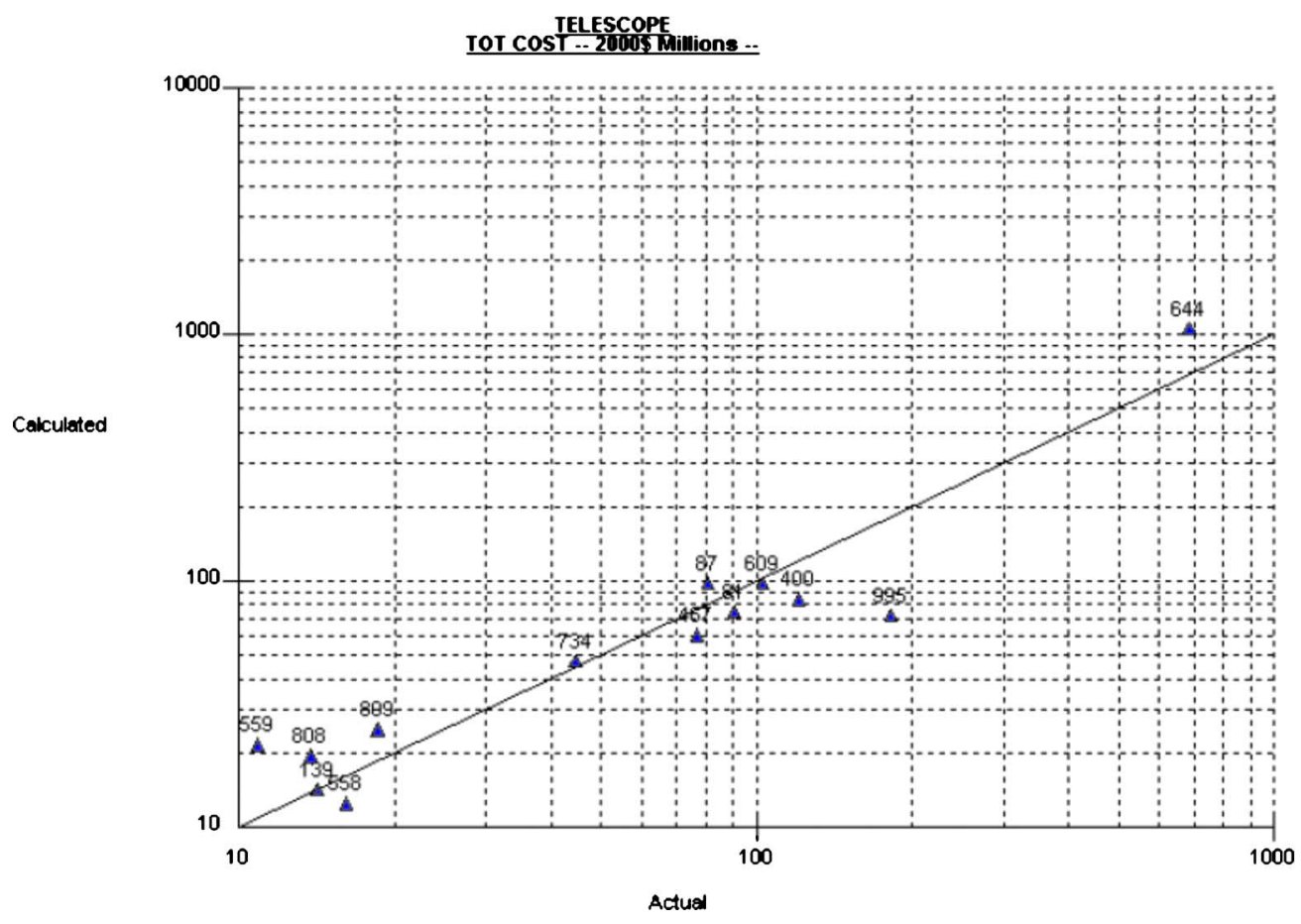

Fig. 2 Estimated cost versus actual cost of Smart Model for 13 space telescope missions. ${ }^{15}$

\subsubsection{Wong cost model}

In 1991, Wong of TRW contributed a chapter on cost modeling to Space and Mission Analysis and Design by Larson and Wertz. ${ }^{16}$ The Wong model presents a two-tier cost breakdown structure for unmanned spacecraft space segment CERs for "research, development, test, and evaluation" (RDTE) cost and "theoretical first unit" (TFU) cost. The chapter states that the model is derived from CERs of two editions of the United States Air Force Unmanned Spacecraft Cost Model. ${ }^{17}$ For optical sensors, the model predicts that cost depends on aperture diameter. For spacecraft and power subsystem CERs, the model estimates cost based on mass and required power. "The advantage of this is that during the concept and mission design phase, weight and power estimates are typically available. The disadvantage is that other parameters that should provide better cost estimates are not included." Additionally, Wong reports multipliers or "wrap" factors for program level costs, technology insertion, and heritage reuse. It is important to note that the author assumes that all technologies involved are "proven" to "at least the component breadboard" level in a relevant environment (i.e., TRL-5/6) before the start of RDTE.

The Wong model (Table 1) estimates that both the RDTE and the TFU costs, for either visible or IR sensors, varies by the sensor aperture diameter to the power of 0.562. This is similar to the 1985 PRC model's power term for infrared systems. And, while PRC reports that the flight unit is 25 to $33 \%$ of the development cost (depending on system wavelength), Wong reports that TFU cost is approximately $40 \%$ of RDTE cost. Also, Wong reports that IR systems cost 2.77 times more than visible sensor systems. While no discussion was offered regarding these ratios, it is this author's opinion that, given the payload applications and the state of technology in the mid to late 1980s, the ratio is probably driven by differences between visible and infrared optical components (i.e., substrate material costs and difficulty to manufacture) and detector costs (i.e., visible arrays versus infrared arrays or scanners). Finally, Wong provides wrap factors for estimating the cost of ground support equipment (GSE) at $11 \%$ of the RTDE and TFU cost, and program level support (i.e., management,

Table 1 Summary of Wong model unmanned spacecraft sensor CERs.

\begin{tabular}{lccccr}
\hline \hline \multicolumn{1}{c}{ Payload } & Range & RDT\&E & Error & TFU & Error \\
\hline IR sensor & 0.2 to $1.2 \mathrm{~m}$ & $3.07 D^{0.562}$ & \pm 0.46 & $1.23 D^{0.562}$ & \pm 0.18 \\
Visible Sensor & 0.2 to $1.2 \mathrm{~m}$ & $1.11 D^{0.562}$ & \pm 0.17 & $0.44 D^{0.562}$ & \pm 0.07 \\
GSE & & $11 \%$ (RDT\&E+TFU) & \pm 0.05 & & \\
Programmatic & & $36 \%$ hardware & \pm 0.08 & $33 \%$ Hardware & \pm 0.03 \\
\hline \hline
\end{tabular}


systems engineering, product assurance, and system test and evaluation) at 33 to $36 \%$ of the hardware cost.

While Wong does report values for the one sigma standard error of the CERs, the only discussion regarding the database that supports this model is that the CERs are derived from historical data for sensor diameters ranging from 0.2 to $1.2 \mathrm{~m}$. There is no discussion of how many or even what sensors are included in the database. Wong does caution the reader that these CERs should not be extended to systems beyond the data range.

Additionally, Wong offers an interesting discussion of the cost impact of inserting new technology into a program verses using heritage technology. While current thinking assumes that insertion of new technology lowers programmatic cost, Wong asserts that new technology increases RDTE and TFU cost by 25 to $100 \%$. An example provided is the cost to change structure designs (of the late 1980s) to graphite epoxy. By comparison, the author states that reuse of heritage technology requiring only moderate modifications can reduce RTDE cost by 40 to $60 \%$, and that reuse of an existing design can reduce RTDE cost by 70 to $90 \%$. Finally, Wong states that because of requirement creep and risk-averse government oversight, the RTDE cost for commercial space systems is only $80 \%$ as much as government spacecraft.

\subsubsection{Horak cost models}

Of all the historical cost models, the Horak model is the most detailed and best documented. The complete model was published via two reports in $1993^{12}$ and $1994 .^{18}$ An updated model was published in $1996 .{ }^{19}$ The purpose of the model was to estimate the total cost of IR sensor payloads operating in geosynchronous and nongeosynchronous orbits or on aircraft. The database consisted of strategic and experimental IR sensor programs (Table 2) which may or may not have actually flown. The developed cost methodology consisted of seven cost estimating relationships (CERs) that estimate the costs of IR sensor assemblies (i.e., optical telescope assembly/structure, focal plane arrays, etc.). The study also developed a CER for integration, assembly, and calibration of the subsystems into a complete system.

The 1993 study developed CERs to estimate the manufacturing cost of the first flight unit of seven different IR sensor subsystems, including the optical telescope assembly/structure. If a qualification unit was developed, its cost was considered as nonrecurring and was included in the developing engineering costs. The manufacturing cost included fabrication, assembly, inspection, and test of the hardware subsystem and did not include any program level costs (e.g., system engineering, program management, etc.). Cost of money, G\&A, and fee for the subsystem component are included. The OTA subsystem includes the telescope optics, mounting hardware, and optical bench support structure. It also included baffles, shroud, and any structure associated with housing the telescope, cryostat, and shroud. Figure 3 shows the derived CER and its statistics for the OTA subsystem. The CER is a multiparametric model consistent with engineering judgment. Horak found that the cost of an OTA increases with mirror diameter and with the number of powered optics in the OTA. And, that the shorter the operating wavelength or the colder the op-
Table 2 Horak cost model database of strategic and experimental IR sensor programs used in the development of CERs for optical telescope assembly/structure.

Homing Overlay Experiment (HOE)

Forward Acquisition Sensor (FAS)

Airborne Optical Adjunct (AOA)

Optical Airborne Measurements Program (OAMP)

Anti-Satellite Program (ASAT)

Teal Ruby Experiment (TRE)

Defense Support Program (DSP 14-17)

Landsat Thematic Mapper (TM-4, TM-5, TM-6)

Infrared Astronomical Satellite (IRAS)

Hubble Space Telescope Optical Telescope Assembly (OTA)

Space Infrared Experiment (SIRE)

High Endo-atmospheric Defense Interceptor (HEDI)

Exo-atmospheric Reentry Intercept System (ERIS)

Ground-based Surveillance \& Tracking System (GSTS)

Midcourse Sensor Experiment (MSX)

Spectrographic Imaging Reflective Telescope (SPIRIT III-MSX)

Space Based Visible Sensor (SBVS-MSX)

erating temperature, the higher the OTA costs. Horak also found that technology investment over time lowers cost. Additionally, the Horak model included multiplier factors that account for fabrication difficulty, e.g., on-axis designs are less expensive than off-axis designs; or, light-weight mirrors cost more to fabricate than solid mirrors. For anyone who has ever tried to align an off-axis telescope or to grind and polish an extremely light-weight mirror, these multiplier factors may seem obvious. But you would be surprised at how many mission planners assume that there is no cost penalty associated with demanding design requirements. The 1993 Horak OTA cost model had a regression of $R^{2}=97.8 \%$, which means that the CER explained $97.8 \%$ of the cost variation of the 16 telescopes in the study.

The 1993 study also developed a CER for the labor and material costs associated with integrating and assembling the subsystem hardware into the complete system. With a regression $R^{2}=96.6 \%$, the study found that:

integration and assembly cost

$=27 \%$ of the total subsystem hardware costs.

While the comparison might be incorrect, Wong estimates that GSE cost and system test and evaluation wrap is approximately $18 \%$ of the total hardware cost. 


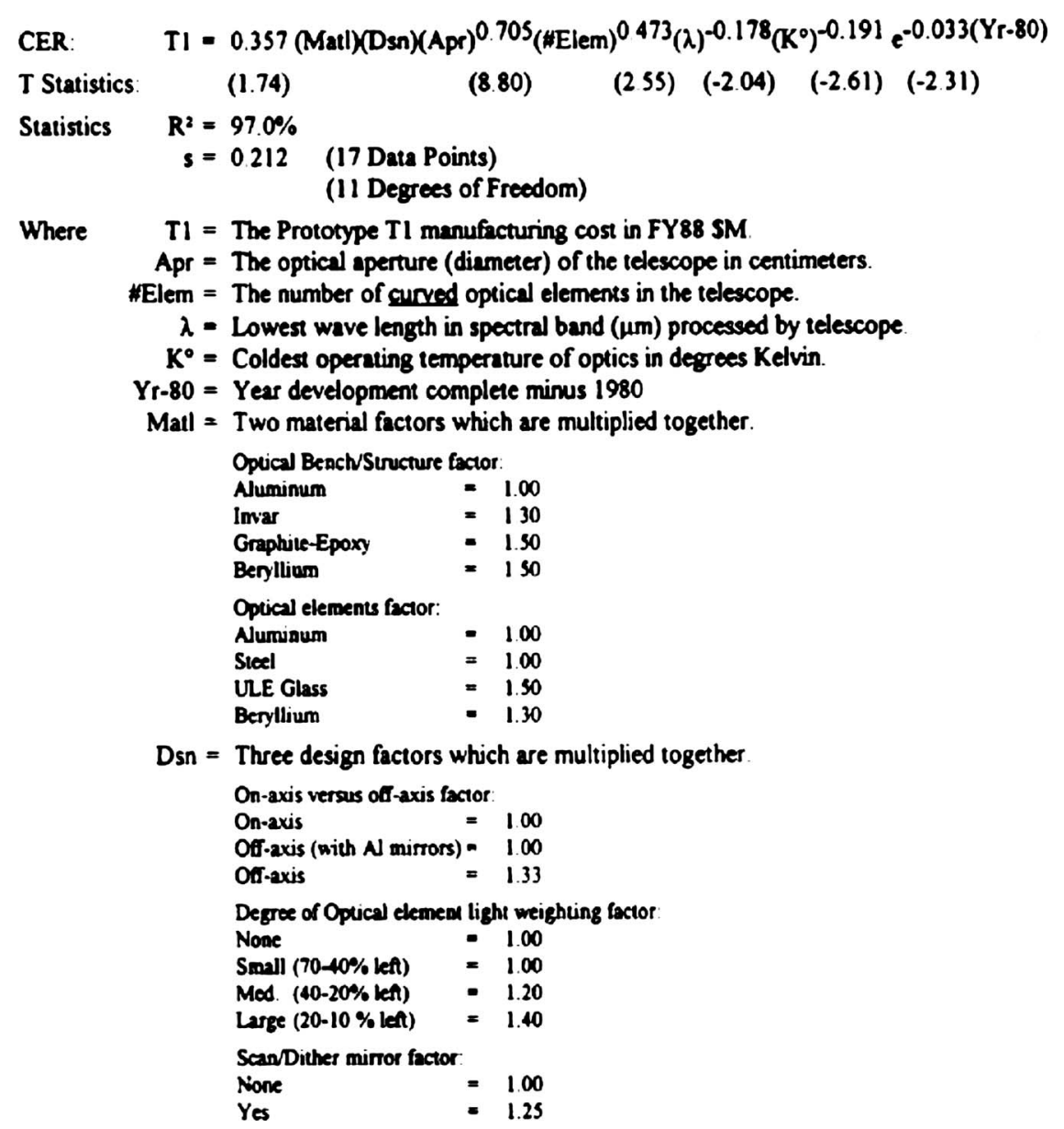

Fig. 3 Horak 1993 cost estimating relationship for optical telescope assembly/structure.

Horak's 1994 report developed CERs to estimate the nonrecurring development engineering costs for the demonstration and validation (D\&V) and the engineering manufacturing development (EMD) phases of a program. With a regression $R^{2}=97.8 \%$, the study found that:

design cost of the OTA $=125 \%$ of the manufacturing

cost of the first flight unit.

This result is interesting in its disagreement with both the PRC and Wong models, which estimate that the design cost will range from 250 to $400 \%$ the cost to manufacture the first flight unit. As expected, Horak found that if more than one flight unit is manufactured, then the design cost increases with quantity, but not linearly. The model assumes a learning curve of $76 \%$.

design cost of the OTA $=125 \%$

$$
\begin{aligned}
& \times \text { (cost of the first flight unit) } \\
& \times \text { (number of flight units })^{0.613} \text {. }
\end{aligned}
$$

The breadboard or engineering unit was found to cost $68 \%$ of the manufacturing cost of the first flight unit, and the qualification unit was found to cost $106 \%$ of the manufacturing cost of the first flight unit.
In 1996, Horak published a set of charts with a multivariable parametric cost model for optical telescope assembly, ${ }^{19}$ which combined design, programmatic, and manufacturing costs (Fig. 4). The parameters were similar to the 1993 model, but the scaling power factors were slightly different. Unfortunately, there is no commentary discussion in the report to explain the details of this model.

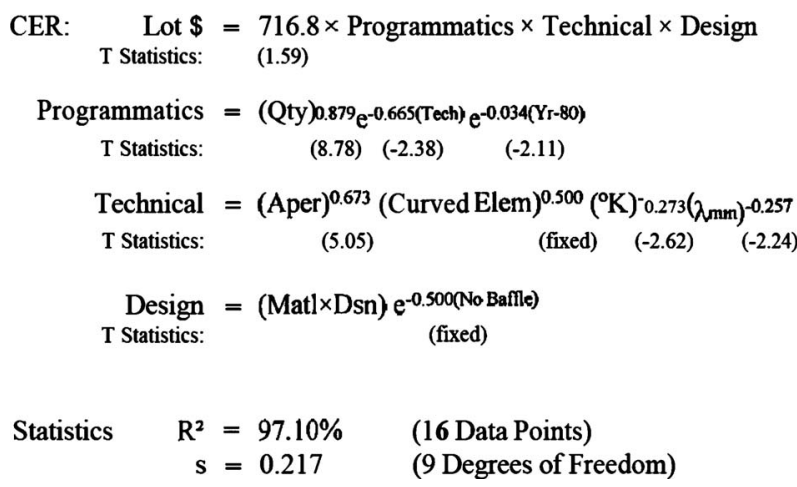

Fig. 4 Horak 1996 cost estimating relationship for optical telescope assembly. 


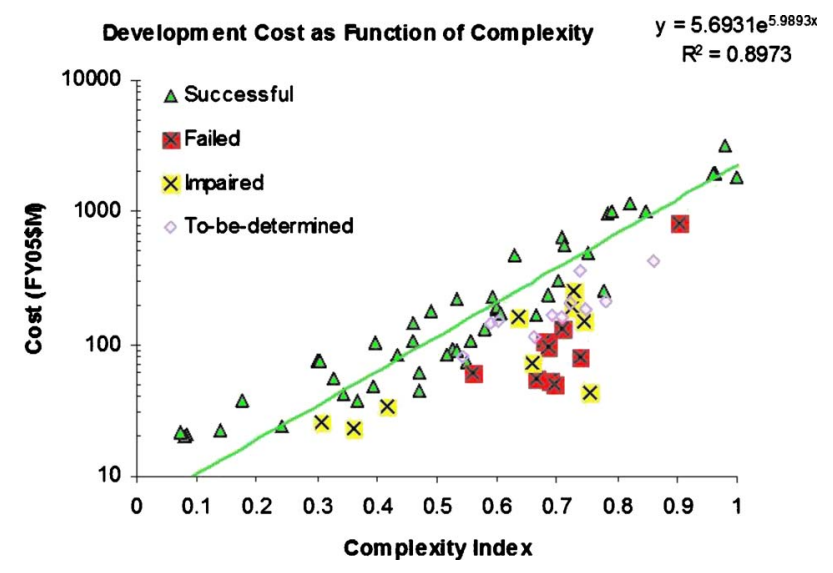

Fig. 5 The more complex a mission concept, the more expensive the mission is to implement (graphic used with permission of Bearden). ${ }^{23}$

\subsubsection{Aerospace cost models}

The Aerospace Corporation (El Segundo, California) has published several papers on cost modeling. ${ }^{20-23}$ While none of these papers are specifically related to space telescopes, they are none the less relevant and illuminative. According to Beardon, there is a direct correlation between mission payload complexity and mission cost, as well as schedule growth. The more complex a mission is, the greater its cost, schedule, and mass is likely to grow. Additionally, the greatest predictor of a mission's success is its technology maturity. The reason for this correlation is because the only way to achieve increasingly demanding performance requirements in a mass and volume constrained launch vehicle is to design increasingly complex mission payload architectures. This cost versus complexity relationship can be seen in Fig. 5, which plots actual mission development cost as a function of the complexity index developed by Beardon. According to the references (and private communication with Beardon), complexity index is determined via a methodology proprietary to the Aerospace Corporation. Specific mission technology or component specifications are compared to similar technologies or components of 150 satellite missions contained in the Aerospace Corporation Complexity Based Risk Assessment (CoBRA) database. ${ }^{23}$ For a detailed discussion of the mission payload complexity index, as well as a full explanation of Fig. 5, the author recommends that readers consult Refs. 20-23.

Similar evidence of a cost versus complexity relationship can be found in the NASA Advanced Mission Cost Model $^{13}$ (Fig. 1), which is typically used to justify (possibly incorrectly) that mass is the dominant mission cost driver. A closer look at the model indicates that difficulty level is a larger cost driver than mass. Unfortunately, this author has no insight into how NASA derived this model or defines difficulty level.

\section{Conclusion}

Parametric cost models are important tools for mission planners. They identify major architectural cost drivers, allow high-level design trades, enable cost-benefit analysis for technology development investment, and provide a basis for estimating total project cost. They can provide guid- ance for expected design costs relative to manufacturing costs; system integration and test costs; or program management overhead. The problem is that all cost models are wrong. They are limited by their database sample sets and assumptions. For example, any model derived from ground-based telescopes is wrong. Similarly, any model based primarily on aircraft sensors or sensor design studies will underestimate the cost of space telescopes. Furthermore, any model that simply says that cost depends solely on mass without any engineering detail might lead one to actually increase total mission cost risk by selecting an unnecessarily complex mission concept or basing a concept on immature unproven technology. It is this author's opinion that any correlation between mass and space telescope cost is a coincidence. Finally, some cost models include programmatic and system engineering costs, while others estimate these as percentage wraps.

As shown in this survey of published cost models, there is no definitive model for the cost of space telescopes. For example, the scaling factor that relates space telescope cost to primary mirror diameter ranges by an order of magnitude from 2.7 (Meinel) to 0.27 (PRC). Of all the historical models surveyed, it is this author's opinion that the one which is closest to "truth" is Smart's multivariable parametric cost model: ${ }^{15}$

cost

$$
=\$ 521.967 M * M D^{1.120} * T R L^{-0.881} * A P^{0.187} * Y T^{-0.330} .
$$

A key subtley of this model is that it is based entirely on NASA space telescopes. What is universally indicated by all the models surveyed in this work is that the more complex the design, the more difficult it will be to build and the more it will cost. Conversely, it is uniformly agreed that the higher the maturity of the technology being implemented, the lower the mission will cost. Some secondary predictions made by the models that require testing are that the cost to develop a mission is 1.25 to $4 \times$ the cost of the flight hardware. And, that infrared telescopes cost two to three times more than visible telescopes.

The bottom line is that blindly using an incorrect and unjustifiable cost estimating relationship without understanding its assumptions and limitations will lead to wrong conclusions and a potentially very expensive decision. Therefore, it is the responsibility of anyone who is reviewing potential mission proposals or selecting missions for flight to perform their due diligence and fully understand the limitations that constrain their foundational assumptions.

\section{References}

1. H. P. Stahl, G. Holmes Rowell, G. Reese, and A. Byberg, "Multivariable parametric cost model for ground optical telescope assembly," Opt. Eng. 44(8), 083001 (2005).

2. A. B. Meinel, "Cost-scaling laws applicable to very large telescopes," Opt. Eng. 18(6), 645-647 (1979).

3. A. B. Meinel, "Cost relationships for nonconventional telescope structural configurations," J. Opt. Soc. Am. 2(1), 14-20 (1981).

4. A. B. Meinel and M. P. Meinel, "Optical phased array configuration for an extremely large telescope," Appl. Opt. 43(3), 601-607 (2004).

5. P. Y. Bely, "Large space optics," NGST Sci. Technol. Expo., ASP Conf. Ser. 207, 34-35 (2000).

6. L. Stepp, L. Daggert, and P. Gillett, "Estimating the costs of extremely large telescopes," Proc. SPIE 4840, 309-321 (2002). 
7. H. P. Stahl, K. Stephens, T. Henrichs, C. Smart, and F. A. Prince, "Single variable parametric cost models for space telescopes," Opt. Eng. 49 (2010) (in press).

8. A. B. Meinel and M. Meinel, "Very large telescopes," Optics News 12(3), 9-14 (Mar. 1986).

9. T. Schmidt-Kaler and P. Rucks, "Telescope costs and cost reduction," Proc. SPIE 2871, 635-640 (1997).

10. G. T. Bellea A. B. Meinel, and M. P. Meinel, "The scaling relationship between telescope cost and aperture size for very large telescopes," Proc. SPIE 5489, 563-570 (2004).

11. P. Y. Bely, The Design and Construction of Large Optical Telescopes, p. 79, Springer-Verlag, New York (2003).

12. J. A. Horak, C. J. Brown, L. M. Giegerich, and W. E. Waller, "Strategic and experimental IR sensor cost model II," Report TR-9114-01, Technomics, Inc. (1993).

13. NASA Advanced Mission Cost Model, see http://cost.jsc.nasa.gov/ AMCM.html

14. PRC Systems Services, "Cost estimating relationships for spaceborne telescopes," PRC D-2241-H Contract 956075, Plantation, FL (1985).

15. C. B. Smart, "Telescope cost estimating relationship," Litton PRC white paper, Plantation, FL (Dec 13 2000).

16. R. Wong, "Cost modeling," Chap. 20 in Space Mission Analysis and Design, 2nd ed., Larson and Wertz, Eds., Microcosm and Kluwer Academic Publishers, New York, (1992).

17. F. Fong, et al., Space Division Unmanned Spacecraft Cost Model, 5th ed., USAF Space Division, Directorate of Cost Analysis, El Segundo, CA (1981); P. Hillebrand, et al., Space Division Unmanned Spacecraft Cost Model, 6th ed., USAF Space Division, Directorate of Cost Analysis, El Segundo, CA (1988).

18. J. A. Horak, C. J. Brown, and W. E. Waller, "Strategic and experimental IR sensor cost model II," Report TR-9311-01, Technomics, Inc. (1994)

19. J. A. Horak, "Cost trends of space electronics," Report 681-0001, Technomics, Inc., (1996)

20. D. A. Bearden, "A complexity-based risk assessment of low-cost planetary missions: when is a mission too fast and too cheap?," 4th IAA Intl. Conf. Low-Cost Planetary Missions, JHU/APL, Laurel, MD, 2-5 May 2000.

21. R. Bitten, D. Emmons, and C. Freaner, "Using historical NASA cost and schedule growth to set future programs and project reserve guide- lines," IEEE Aerospace Conference, Big Sky, MT, March 3-10, 2007. 22. C. W. Freaner, et al., "An assessment of the inherent optimism in early conceptual designs and its effect on cost and schedule growth," SSCAG/SCAF/EACE 2009 Joint International Conference, ESTEC, Noordwijk, The Netherlands, 15-16 May 2008.

23. D. A. Bearden, "Perspectives on NASA mission cost and schedule performance trends," GSFC System Engineering Symp. NASA, 3 June 2008.

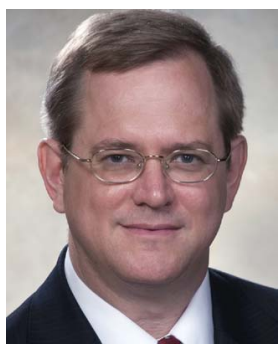

H. Philip Stahl is a Senior Optical Physicist at NASA MSFC. He is the James Webb Space Telescope (JWST) Optical Components Technical Lead. Previously, he was a senior staff optical engineer at Raytheon Danbury (formerly Hughes Danbury Optical Systems, now Goodrich Aerospace) where he was the lead optical engineer for the 4 meter LAMP mirror. As President of Stah Optical Systems Inc, he supported several NASA Space Shuttle microgravity experiments. Also, he was an assistant professor of physics and applied optics at Rose-Hulman Institute of Technology, the Optical Products Manager at Breault Research Organization (BRO), and a Senior Optical Systems Engineer at BRO. Prior to that, he worked at Perkin-Elmer, Hughes Aircraft, and Wright-Patterson AFB. Finally, he was a Faculty Fellow at NASA Lewis (now Glenn) Research Center. He is a leading authority in optical metrology, optical engineering, and phase-measuring interferometry. Many of the world's largest telescopes have been fabricated with the aid of high-speed and infrared phase-measuring interferometers developed by him including the Keck, VLT and Gemini telescopes. He is a member of OSA, Fellow of SPIE, a past SPIE Director, and current International Commission for Optics (ICO) Vice President. He earned his PhD in Optical Science at the University of Arizona Optical Sciences Center in 1985. 